\title{
A Class of Stochastic Nonlinear Delay System with Jumps
}

\author{
Ling Bai, ${ }^{1}$ Kai Zhang, ${ }^{1}$ and Wenju Zhao' \\ ${ }^{1}$ College of Mathematics, Jilin University, Changchun 130061, China \\ ${ }^{2}$ Florida State University, Department of Scientific Computing, Tallahassee, FL 32306, USA \\ Correspondence should be addressed to Ling Bai; bailing@jlu.edu.cn and Kai Zhang; kzhang@jlu.edu.cn
}

Received 30 August 2013; Revised 25 November 2013; Accepted 9 December 2013; Published 30 January 2014

Academic Editor: Chong Lin

Copyright (C) 2014 Ling Bai et al. This is an open access article distributed under the Creative Commons Attribution License, which permits unrestricted use, distribution, and reproduction in any medium, provided the original work is properly cited.

\begin{abstract}
We consider stochastic suppression and stabilization for nonlinear delay differential system. The system is assumed to satisfy local Lipschitz condition and one-side polynomial growth condition. Since the system may explode in a finite time, we stochastically perturb this system by introducing independent Brownian noises and Lévy noise feedbacks. The contributions of this paper are as follows. (a) We show that Brownian noises or Lévy noise may suppress potential explosion of the solution for some appropriate parameters. (b) Using the exponential martingale inequality with jumps, we discuss the fact that the sample Lyapunov exponent is nonpositive. (c) Considering linear Lévy processes, by the strong law of large number for local martingale, sufficient conditions for a.s. exponentially stability are investigated in Theorem 13.
\end{abstract}

\section{Introduction}

Usually, for a given nonlinear system

$$
\begin{aligned}
& \dot{x}(t)=f(x(t), t) \text { or delay system } \\
& \dot{x}(t)=f(x(t), x(t-\delta(t)), t),
\end{aligned}
$$

$f$ satisfies the polynomial growth condition; the solutions may explode in finite time. It is well know that noises can stabilize the given unstable system or make this system even more stable if it is already stable. To stabilize the system, the authors in [1] introduced a polynomial Brownian noise to suppress potential explosion and then considered another linear Brownian noise to stabilize the suppressed equation. For more details, refer to [1-3].

For example, considering a simple logistic equation

$$
\dot{x}(t)=x(1+x),
$$

with initial value $x(0)=1$, the expression of the solution is $x(t)=1 /\left(-1+2 e^{-t}\right)$, and there has been only a local solution for $1 \leq t \leq \log 2$; that is, $x(t)$ will explode and the explosion time is $\tau_{e}=\log 2$. To suppress explosion and stabilize system (2), the dependent scalar Brownian noises are introduced in [1] and they disturbed system (2) into

$$
\dot{x}(t)=x(t)[1+x(t)] d t+2 x(t) d W_{1}(t)+x^{2}(t) d W_{2}(t) ;
$$

the theoretical proof and simulations show that noises may not only suppress explosion of solution (3), but also stabilize unstable system (2).

However, all of the papers mentioned above only considered the perturbation by Brownian noises. Recently, there has been increasing attention devoted to the effect of different noises. Non-Gaussian random processes also play an important role in modelling stochastic dynamics. Typical examples of non-Gaussian stochastic processes are Lévy processes and processes arising from Poisson random measures. Further we assume that the different stochastic processes are independent.

Motivated by the previous articles [1-3], we will study the following delay SDE with jumps:

$$
\begin{aligned}
d x(t)= & f(x(t), x(t-\tau), t) d t+\sigma|x(t)|^{\beta} x(t) d W_{1}(t) \\
& +q|x(t-\tau)|^{\beta^{\prime}} x(t) d W_{2}(t) \\
& +x(t) \int_{\mathbb{Y}} H\left(x\left(t^{-}\right), x(t-\tau)^{-}, u\right) \widetilde{N}(d t, d u),
\end{aligned}
$$

with initial data

$$
\{x(t):-\tau \leq t \leq 0\}=\left\{\xi(t):-\tau \leq t \leq 0 \in C_{\mathscr{F}_{0}}^{b}([-\tau, 0])\right\} .
$$


Here $W_{i}(t)(i=1,2)$ is defined on the probability space, $\left(\Omega, \mathscr{F},\left\{\mathscr{F}_{t}\right\}_{t \geq 0}, P\right)$ being a complete probability space with a filtration $\left\{\mathscr{F}_{t}\right\}_{t \geq 0}$ satisfying the usual conditions (i.e., it is right continuous and $\mathscr{F}_{0}$ contains all $P$-null sets $) . N(d t, d u)$ is realvalued Poisson counting measure with characteristic measure $\lambda$ on a measurable subset $\mathbb{Y}$ of $[0, \infty)$ with $\lambda(\mathbb{Y})<\infty$, $\widetilde{N}(d t, d u)=N(d t, d u)-\lambda(d u) d t . W_{i}(t)$ is the 1-dimensional standard Brownian motion with $W_{i}(0)=0$. We assume that $W_{i}(t)$ and Poisson process $N$ are independent. Let $\tau>0$ and $C\left([-\tau, 0] ; R^{n}\right)$ denote the family of continuous function $\phi$ from $[-\tau, 0]$ to $R^{n}$, which is a Banach space with the norm $\|\phi\|=\sup _{-\tau \leq \theta \leq 0}|\phi(\theta)|$. Denote by $C_{\mathscr{F}_{0}}^{b}\left([-\tau, 0] ; R^{n}\right)$ the family of all bounded, $\mathscr{F}_{0}$-measurable, $C\left([-\tau, 0] ; R^{n}\right)$-valued random variable.

We will impose the following assumptions on $f: R^{n} \times$ $R^{n} \times R_{+} \rightarrow R^{n}$ as local Lipschitz continuous and satisfy the polynomial growth condition.

Assumption 1. For any $t \geq 0, u \in \mathbb{Y}, x, y \in R^{n}$, and $H(x, y, u)>-1$. For each integer $k \geq 1, \ldots$, there is a positive number $L_{k}$ such that

$$
\begin{aligned}
& \left|f\left(x_{1}, y_{1}, t\right)-f\left(x_{2}, y_{2}, t\right)\right|^{2} \\
& \quad+\int_{\mathbb{Y}}\left|x_{1} H\left(x_{1}, y_{1}, u\right)-x_{2} H\left(x_{2}, y_{2}, u\right)\right|^{2} \lambda(d u) \\
& \quad \leq L_{k}\left(\left|x_{1}-x_{2}\right|^{2}+\left|y_{1}-y_{2}\right|^{2}\right),
\end{aligned}
$$

for all $t \geq 0$ and those $x_{1}, x_{2}, y_{1}, y_{2} \in R^{n}$ with $\left|x_{1}\right| \vee\left|x_{2}\right| \vee$ $\left|y_{1}\right| \vee\left|y_{2}\right| \leq k$

Assumption 2. There are some nonnegative numbers $\alpha, \kappa, \bar{\kappa}$, and $\gamma$ such that

$$
\langle x, f(x, y, t)\rangle \leq|x|^{2}\left(\kappa|x|^{\alpha}+\bar{\kappa}|y|^{\alpha}+\gamma\right)
$$

for all $(x, y, t) \in R^{n} \times R^{n} \times R_{+}$.

In reference to the existing results in the literature, our contributions are as follows.

(i) Use stochastic delay differential equation with jump diffusion to model the evolutions of nonlinear dynamics.

(ii) Discuss the global solution of the stochastic equation under polynomial growth condition; in particular, we compare the effect of suppression solution of different type of noises.

(iii) For stabilization of noise, we can verify that the linear jump process has an effect on a.s. exponential stability.

To the best of our knowledge, under the assumption of polynomial growth condition, analysis of nonlinear stochastic system with jumps has not been fully investigated and few results have been available so far. We aim to discuss the question in this work.

The organization of the paper is as follows. Section 2 shows the global solution of system (4), which indicates that Brownian noises or Lévy noise may suppress potential explosion of the solution for some appropriate parameters. The results of Section 3 guarantee that SDE (4) is stochastically ultimate boundedness. Our main results emerge in Section 4. We discuss the sample Lyapunov exponent is nonpositive and reveal the stabilization effect of Lévy noise. Conclusions and extensions are made in Section 5.

\section{Global Solution}

Now we first prove the existence of the global solution to (4).

Theorem 3. Under the conditions of Assumptions 1 and 2, for any given initial data $\left\{\xi(t):-\tau \leq t \leq 0 \in C_{\mathscr{F}_{0}}^{b}([-\tau, 0])\right\}$, supposing that $2 \beta>\max \left\{\alpha, 2 \beta^{\prime}\right\}$ and $\sigma \neq 0$, there a.s. exists a unique global solution $x(t)$ to $(4)$ on $t \in[-\tau, \infty)$.

Proof. Since both the drift term and the diffusion terms of (4) are locally Lipschitz, by similar standard truncated argument $[4,5]$, there is a unique local maximal solution $x(t)$ on $t \in$ $\left[-\tau, \tau_{e}\right)$, where $\tau_{e}$ is the explosion time defined by

$$
\tau_{e}=: \inf \{t>0:|x(t)|=\infty\} .
$$

To show that this solution is actually global, we need to prove that $\tau_{e}=\infty$ a.s.

Let $m_{0}$ be a sufficiently large positive number such that $|\xi(0)| \leq m_{0}$. For each $m \geq m_{0}$, we define the stopping time

$$
\tau_{m}=\inf \left\{t \in\left[0, \tau_{e}\right):|x(t)|>m\right\} .
$$

Clearly $\tau_{m}$ is increasing as $m \rightarrow \infty$ and $\lim _{m \rightarrow \infty} \tau_{m}=: \tau_{\infty} \leq$ $\tau_{e}$; if we can obtain that $\tau_{\infty}=\infty$ a.s., then $\tau_{e}=\infty$ a.s.; that is, to complete the proof, all we need to show is that $\tau_{\infty}=\infty$ a.s.; this is also equivalent to proving that, for any $t>0, P\left(\tau_{m} \leq\right.$ $t) \rightarrow 0$ as $m \rightarrow \infty$. For $p \in(0,1)$, define a $C^{2}$-function: $R^{n} \times \mathbb{S} \rightarrow \bar{R}_{+}$by

$$
V(x)=|x(t)|^{p}
$$

If, for any $m>0$ and $|x|>m$, one can apply Itô formula to compute that

$$
\begin{aligned}
d V(x)= & L V(x, y) d t+p \sigma|x(t)|^{\beta+p} d W_{1}(t) \\
& +p q|x(t)|^{p}|x(t-\tau)|^{\beta^{\prime}} d W_{2}(t) \\
& +\int_{\mathbb{Y}}|x|^{p}\left[(1+H(x, y, u))^{p}-1\right] \widetilde{N}(d t, d u),
\end{aligned}
$$

where $y(t)=x(t-\tau)$ and $L V$ is defined as

$$
\begin{aligned}
& L V(x, y) \\
& =p|x|^{p-2}\langle x, f(x, y, t)\rangle+\frac{p(p-1)}{2} q^{2}|x|^{p}|y|^{2 \beta^{\prime}} \\
& \quad+\frac{p(p-1)}{2} \sigma^{2}|x|^{2 \beta+p} \\
& \quad+\int_{\mathbb{Y}}\left\{|x|^{p}\left[|1+H(x, y, u)|^{p}-1-p H(x, y, u)\right]\right\} \lambda(d u),
\end{aligned}
$$


therefore we get from Dynkin's formula [6], taking expectation from two sides of (11)

$\mathbb{E} V\left(x\left(\tau_{m} \wedge T\right)\right)=\mathbb{E} V(\xi(0))+\mathbb{E} \int_{0}^{\tau_{m} \wedge T} L V(x(s), y(s)) d s$.

Using the inequality $x^{r} \leq 1+r(x-1), x \geq 0,0 \leq r \leq 1$, we have

$$
(1+H(x, y, u))^{p}-1-p H(x, y, u) \leq 0
$$

because $H(x, y, u)>-1$.

Combining (11) and (14) and recalling the well-known Young inequality, we therefore have

$$
\begin{aligned}
L V(x, y) \leq & p|x|^{p-2}|x|^{2}\left(\kappa|x|^{\alpha}+\bar{\kappa}|y|^{\alpha}+\gamma\right) \\
& +\frac{p(p-1)}{2} q^{2}|x|^{p}|y|^{2 \beta^{\prime}}+\frac{p(p-1)}{2} \sigma^{2}|x|^{2 \beta+p} \\
\leq & \frac{p(p-1)}{2} \sigma^{2}|x|^{2 \beta+p}+p \kappa|x|^{\alpha+p}+p \bar{\kappa}|x|^{p}|y|^{\alpha} \\
& +p \gamma|x|^{p}+q^{2}|x|^{p}|y|^{2 \beta^{\prime}} \\
\leq & \frac{p(p-1)}{2} \sigma^{2}|x|^{2 \beta+p}+p\left(\kappa+\frac{\bar{\kappa} p}{\alpha+p}\right)|x|^{\alpha+p} \\
& +\frac{p q^{2}}{2 \beta^{\prime}+p}|x|^{2 \beta^{\prime}+p} \\
& +\frac{2 q^{2} \beta^{\prime}}{2 \beta^{\prime}+p}|y|^{2 \beta^{\prime}+p}+p \frac{\bar{\kappa} \alpha}{\alpha+p}|y|^{\alpha+p}+p \gamma|x|^{p}
\end{aligned}
$$

then we have from (15) that

$$
\begin{aligned}
\mathbb{E} V & \left(x\left(\tau_{m} \wedge T\right)\right) \\
= & V(\xi(0))+\mathbb{E} \int_{0}^{\tau_{m} \wedge T} N(x(s)) d s \\
& +\frac{\alpha \bar{\kappa} p}{\alpha+p} \mathbb{E} \int_{0}^{\tau_{m} \wedge T}\left[|x(s-\tau)|^{\alpha+p}-|x(s)|^{\alpha+p}\right] d s \\
& +\frac{2 q^{2} \beta^{\prime}}{2 \beta^{\prime}+p} \mathbb{E} \int_{0}^{\tau_{m} \wedge T}\left[|x(s-\tau)|^{2 \beta^{\prime}+p}-|x(s)|^{2 \beta^{\prime}+p}\right] d s,
\end{aligned}
$$

where

$$
\begin{aligned}
\int_{0}^{\tau_{m} \wedge T} & {\left[|x(s-\tau)|^{\alpha+p}-|x(s)|^{\alpha+p}\right] d s } \\
\leq & \int_{-\tau}^{\tau_{m} \wedge T-\tau}|x(s)|^{\alpha+p} d s-\int_{0}^{\tau_{m} \wedge T}|x(s)|^{\alpha+p} d s \\
\leq & \int_{-\tau}^{\tau_{m} \wedge T}|x(s)|^{\alpha+p} d s-\int_{0}^{\tau_{m} \wedge T}|x(s)|^{\alpha+p} d s \\
\leq & \int_{-\tau}^{0}|\xi(s)|^{\alpha+p} d s<+\infty, \\
\int_{0}^{\tau_{m} \wedge T} & {\left[|x(s-\tau)|^{2 \beta^{\prime}+p}-|x(s)|^{2 \beta^{\prime}+p}\right] d s } \\
\leq & \int_{-\tau}^{0}|\xi(s)|^{2 \beta^{\prime}+p} d s<+\infty,
\end{aligned}
$$

$$
\begin{aligned}
N(x)= & \frac{p(p-1)}{2} \sigma^{2}|x|^{2 \beta+p}+p(\kappa+\bar{\kappa})|x|^{\alpha+p} \\
& +q^{2}|x|^{2 \beta^{\prime}+p}+p \gamma|x|^{p} .
\end{aligned}
$$

Noting that $p \in(0,1)$, and $2 \beta>\max \left\{\alpha, 2 \beta^{\prime}\right\}$, by the boundedness of polynomial function $N(x)$, there is a constant $\bar{N}$ such that $N(x) \leq \bar{N}$. Consider

$$
\begin{aligned}
\mathbb{E} V\left(x\left(\tau_{m} \wedge T\right)\right) & \\
\leq & V(\xi(0))+\bar{N} T+\frac{\alpha \bar{\kappa} p}{\alpha+p} \int_{-\tau}^{0}|\xi(s)|^{\alpha+p} d s \\
& +\frac{2 q^{2} \beta^{\prime}}{2 \beta^{\prime}+p} \int_{-\tau}^{0}|\xi(s)|^{2 \beta^{\prime}+p} d s=: N_{T},
\end{aligned}
$$

where $N_{T}$ is independent of $m$.

By the definition of $\tau_{m},\left|x\left(\tau_{m}\right)\right|=m$, let $\Omega_{m}=\left\{\tau_{m} \leq T\right\}$ for sufficient large $m$ and for every $\omega \in \Omega_{m}$ such that $x\left(\tau_{m}, \omega\right)$ equals $m$; hence,

$$
\begin{aligned}
P\left(\tau_{m} \leq T\right)\left|x\left(\tau_{m}\right)\right|^{p} & =P\left(\tau_{m} \leq T\right) V\left(x\left(\tau_{m}\right)\right) \\
& \leq \mathbb{E}\left[I_{\left\{\tau_{m} \leq T\right\}} V\left(x\left(\tau_{m} \wedge T\right)\right)\right] \\
& \leq \mathbb{E} V\left(x\left(\tau_{m} \wedge T\right)\right) \leq N_{T} .
\end{aligned}
$$

Letting $m \rightarrow \infty$ implies that

$$
\limsup _{m \rightarrow \infty} P\left(\tau_{m} \leq T\right)=0 .
$$

So we must obtain $\tau_{\infty}=\infty$ a.s., as required. The proof is complete.

The key of this proof in Theorem 3 is the boundedness of $L V(x, y)$, under the conditions of Assumptions 1 and 2 and $2 \beta>\max \left\{\alpha, 2 \beta^{\prime}\right\}$, which imply that the Brownian noise $\sigma(r(t))|x(t)|^{\beta} x(t) d W_{1}(t)$ plays the important role to suppress potential explosion of the solution and guarantees the existence of the global solution.

In what follows, in order to highlight the explosive suppression by jump processes, we will consider the following suitable condition.

Assumption 4. For any $t \geq 0, x \in R^{n}, u \in \mathbb{Y}$, and $H(x, y, u)>$ -1 . If $p \in(0,1)$, there exists $\alpha^{\prime}, \alpha^{\prime \prime}, \delta, \delta_{1}, \delta_{2} \geq 0$ and $\alpha^{\prime}>\alpha^{\prime \prime}$ such that

$$
\begin{aligned}
J(x, y, p) & =: \int_{\mathbb{Y}}\left[|1+H(x, y, u)|^{p}-1-p H(x, y, u)\right] \lambda(d u) \\
& \leq \delta-\delta_{1}|x|^{\alpha^{\prime}}+\delta_{2}|y|^{\alpha^{\prime \prime}} .
\end{aligned}
$$

We still obtain the existence of global solution of (4).

Theorem 5. Under the conditions of Assumptions 1, 2, and 4, if for any given initial data $\left\{\xi(t):-\tau \leq t \leq 0 \in C_{\mathscr{F}_{0}}^{b}([-\tau, 0])\right\}$, $2 \beta \leq \alpha \leq \alpha^{\prime}$, there a.s. exists a unique global solution $x(t)$ to (4) on $t \in[-\tau, \infty)$. 
Proof. Since the proof is similar to that of Theorem 3, we here only sketch the proof to point out the variation between them. Noting (12), (15), and (16), we obtain from Assumption 4 that for $p \in(0,1)$, that

$$
\begin{aligned}
& L V(x, y) \\
& \leq \frac{p(p-1)}{2} \sigma^{2}|x|^{2 \beta+p}+p\left(\kappa+\frac{\bar{\kappa} p}{\alpha+p}\right)|x|^{\alpha+p} \\
& \quad+p \frac{\bar{\kappa} \alpha}{\alpha+p}|y|^{\alpha+p}+p \gamma|x|^{p} \\
& \quad+\int_{\mathbb{Y}}\left\{|x|^{p}\left[|1+H(x, k, u)|^{p}-1-p H(x, k, u)\right]\right\} \lambda(d u) \\
& \leq \\
& \quad \frac{p(p-1)}{2} \sigma^{2}|x|^{2 \beta+p}-\delta_{1}|x|^{\alpha^{\prime}+p}+p\left(\kappa+\frac{\bar{\kappa} p}{\alpha+p}\right)|x|^{\alpha+p} \\
& \quad+\frac{\delta_{2} p}{\alpha^{\prime \prime}+p}|x|^{\alpha^{\prime \prime}+p}+p \frac{\bar{\kappa} \alpha}{\alpha+p}|y|^{\alpha+p} \\
& \quad+\frac{\delta_{2} \alpha^{\prime \prime}}{\alpha^{\prime \prime}+p}|y|^{\alpha^{\prime \prime}+p}+(p \gamma+\delta)|x|^{p} .
\end{aligned}
$$

Therefore, we have

$$
\begin{aligned}
& \mathbb{E} V\left(x\left(\tau_{m} \wedge T\right)\right) \\
& =V(\xi(0))+\mathbb{E} \int_{0}^{\tau_{m} \wedge T} N_{1}(x(s)) d s+\frac{\alpha \bar{\kappa} p}{\alpha+p} \\
& \quad \times \mathbb{E} \int_{0}^{\tau_{m} \wedge T}\left[|x(s-\tau)|^{\alpha+p}-|x(s)|^{\alpha+p}\right] d s \\
& +\frac{\delta_{2} \alpha^{\prime \prime}}{\alpha^{\prime \prime}+p} \mathbb{E} \int_{0}^{\tau_{m} \wedge T}\left[|x(s-\tau)|^{\alpha^{\prime \prime}+p}-|x(s)|^{\alpha^{\prime \prime}+p}\right] d s,
\end{aligned}
$$

where

$$
\begin{aligned}
N_{1}(x)= & \frac{p(p-1)}{2} \sigma^{2}|x|^{2 \beta+p}-\delta_{1}|x|^{\alpha^{\prime}+p} \\
& +p(\kappa+\bar{\kappa})|x|^{\alpha+p}+\delta_{2}|x|^{\alpha^{\prime \prime}+p}+(p \gamma+\delta)|x|^{p} .
\end{aligned}
$$

Noting that $p \in(0,1)$ and $\alpha^{\prime}>\alpha \geq 2 \beta$, by the boundedness of polynomial functions, there is a constant $\bar{N}_{1}$ such that $N_{1}(x) \leq \bar{N}_{1}$,

$$
\begin{aligned}
& \mathbb{E} V\left(x\left(\tau_{m} \wedge T\right)\right) \\
& \leq V(\xi(0))+\bar{N}_{1} T+\frac{\alpha \bar{\kappa} p}{\alpha+p} \int_{-\tau}^{0}|\xi(s)|^{\alpha+p} d s \\
& \quad+\frac{\delta_{2} \alpha^{\prime \prime}}{\alpha^{\prime \prime}+p} \int_{-\tau}^{0}|\xi(s)|^{\alpha^{\prime \prime}+p}=: \bar{N}_{T},
\end{aligned}
$$

where $\bar{N}_{T}$ is independent of $m$.

By $p \in(0,1)$ and $\alpha^{\prime}>\alpha \geq 2 \beta$, the boundedness of $L V(x, y)$ follows; thus, the desired assertion can be carried out the same procedure as the last part of Theorem 3.
Remark 6. Theorem 5 indicates under Assumptions 1, 2, and 4 and $2 \beta \leq \alpha<\alpha^{\prime}$ that jump diffusion process becomes the leading role of suppressing the explosion of the solution.

Therefore, we give two simulations under Theorems 3 and 5. See Figure 1.

\section{Stochastic Ultimate Boundedness}

Theorems 3 and 5 show that the solution of SDE (4) with a given initial data will not explode. This nice property provides us with a great opportunity to discuss how the solution varies in $R^{n}$ in more details. In this section, we will give two definitions and then give sufficient conditions which guarantee SDE (4) is stochastically ultimate boundedness.

Definition 7. The solutions $x(t)$ of SDE (4) are said to be asymptotically bounded in $p$ th moment if there is a positive constant $K_{p}$ such that the solution of SDE (4) with a given initial value has the property that

$$
\limsup _{t \rightarrow \infty} \mathbb{E}|x(t)|^{p} \leq K_{p}
$$

for any initial data $\left\{\xi(t):-\tau \leq t \leq 0 \in C_{\mathscr{F}_{0}}^{b}([-\tau, 0])\right\}$.

Definition 8. SDE (4) is said to be stochastically ultimate boundedness if, for any $\varepsilon \in(0,1)$, there exist positive constants $\chi=\chi(\epsilon)$ such that

$$
\limsup _{t \rightarrow+\infty} P\{|x(t)| \leq \chi\} \geq 1-\epsilon,
$$

where $x(t)$ is the solution of SDE (4) with any positive initial data.

In the light of Markov inequality, it is obvious that if a stochastic equation is $p$ th moment boundedness, its solutions must be stochastically ultimately bounded. So we will begin with the following lemma and make use of it to obtain the stochastically ultimate boundedness of SDE (4).

Lemma 9. Under the conditions of Assumptions 1 and 2, for $p \in(0,1)$, if $2 \beta>\alpha, \sigma \neq 0$, there exists a constant $K_{p}$ such that the global solution $x(t)$ of SDE (4) with any initial data $\left\{\xi(t):-\tau \leq t \leq 0 \in C_{\mathscr{F}_{0}}^{b}([-\tau, 0])\right\}$ has the property that

$$
\limsup _{t \rightarrow \infty} \mathbb{E}\left(|x(t)|^{p}\right) \leq K_{p}
$$

where $K_{p}$ is dependent on $p$ and independent of the initial data.

Proof. First, Theorem 3 indicates that the solution $x(t)$ of (4) will exist for $t \in[-\tau, \infty)$ with probability 1 . For any $\varepsilon>0$ and $p \in(0,1)$, by virtue of Dynkin's formula to $e^{\varepsilon t} V(x)$, we obtain

$$
\begin{aligned}
\mathbb{E} V(x)= & e^{-\varepsilon t} V(\xi(0)) \\
& +e^{-\varepsilon t} \mathbb{E} \int_{0}^{t} e^{\varepsilon s}[L V(x(s), y(s))+\varepsilon V(x(s))] d s .
\end{aligned}
$$




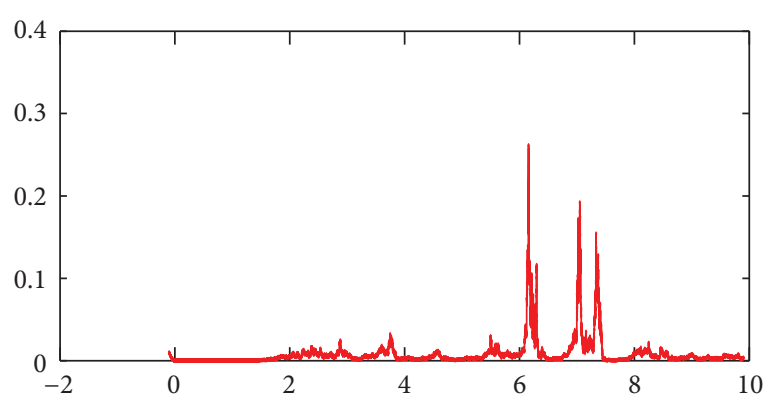

$\longrightarrow$ Parameters satisfy Theorem 3

(a)

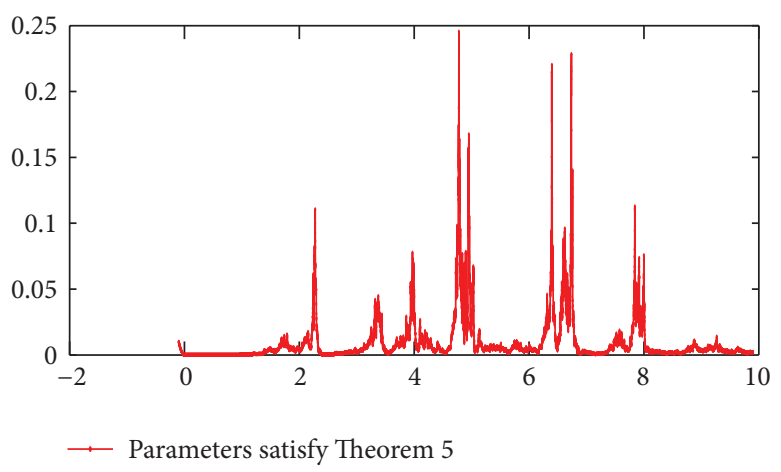

(b)

Figure 1: The simulation solutions of delay stochastic system (4) under the different conditions of Theorems 3 and 5.

Here $L V(x, y)$ is defined as (12). Therefore, by Assumptions 1 and 2, (11)-(15), we have

$$
\begin{aligned}
& L V(x, y)+\varepsilon V(x) \\
& \leq \frac{p(p-1)}{2} \sigma^{2}|x|^{2 \beta+p}+p\left(\kappa+\frac{\bar{\kappa} p}{\alpha+p}\right)|x|^{\alpha+p} \\
& \quad+p \frac{\bar{\kappa} \alpha}{\alpha+p}|y|^{\alpha+p}+(p \gamma+\varepsilon)|x|^{p} .
\end{aligned}
$$

Similar to the proof of Theorem 3, we have from (29) and (30) that

$$
\begin{aligned}
\mathbb{E} V & (x(t)) \\
= & e^{-\varepsilon t} V(\xi(0))+e^{-\varepsilon t} \mathbb{E} \int_{0}^{t} e^{\varepsilon s} N^{*}(x(s)) d s \\
& +e^{-\varepsilon t} \frac{\alpha \bar{\kappa} p}{\alpha+p} \mathbb{E} \int_{0}^{t} e^{\varepsilon s}\left[|x(s-\tau)|^{\alpha+p}-e^{\varepsilon \tau}|x(s)|^{\alpha+p}\right] d s,
\end{aligned}
$$

where

$$
\begin{gathered}
N^{*}(x)=\frac{p(p-1)}{2} \sigma^{2}|x|^{2 \beta+p}+p\left(\kappa+\frac{\bar{\kappa} p}{\alpha+p}\right)|x|^{\alpha+p} \\
+e^{\varepsilon \tau} \frac{p \bar{\kappa} \alpha}{\alpha+p}|x|^{\alpha+p}+(p \gamma+\varepsilon)|x|^{p}, \\
\int_{0}^{t} e^{\varepsilon s}\left[|x(s-\tau)|^{\alpha+p}-e^{\varepsilon \tau}|x(s)|^{\alpha+p}\right] d s \\
\quad=\int_{-\tau}^{t-\tau} e^{\varepsilon(s+\tau)}|x(s)|^{\alpha+p} d s-\int_{0}^{t} e^{\varepsilon(s+\tau)}|x(s)|^{\alpha+p} d s \\
\leq \int_{-\tau}^{0} e^{\varepsilon(s+\tau)}|\xi(s)|^{\alpha+p} d s<\infty .
\end{gathered}
$$

Notice that $\sigma \neq 0$; if $p \in(0,1)$ and $2 \beta>\alpha$, (32) has upper boundedness $N^{*}(x) \leq \bar{N}^{*}$, which means that, combined with (31),

$$
\begin{aligned}
\mathbb{E} V(x) \leq & e^{-\varepsilon t} V(\xi(0))+\frac{\bar{N}^{*}}{\varepsilon}\left(1-e^{-\varepsilon t}\right) \\
& +e^{-\varepsilon t} \frac{\alpha \bar{\kappa} p}{\alpha+p} \int_{-\tau}^{0} e^{\varepsilon(s+\tau)}|\xi(s)|^{\alpha+p} d s .
\end{aligned}
$$

Letting $t \rightarrow \infty$ gives

$$
\limsup _{t \rightarrow \infty} \mathbb{E}|x(t)|^{p} \leq \frac{\bar{N}^{*}}{\varepsilon} .
$$

Lemma 9'. Under the conditions of Assumptions 1, 2, and 4, supposing that $p \in(0,1)$ and $2 \beta \leq \alpha<\alpha^{\prime}$, there exists a constant $K_{p}$ such that the global solution $x(t)$ of SDE (4) with any given positive initial value has the property that

$$
\limsup _{t \rightarrow \infty} \mathbb{E}\left(|x(t)|^{p}\right) \leq K_{p},
$$

where $K_{p}$ is dependent on $p$ and independent of the initial value.

In order to complete the proof, we only show the key step, from (29), combined with (22), which was rewritten as

$$
\begin{aligned}
\mathbb{E} V(x(t)) \\
=e^{-\varepsilon t} V(\xi(0))+e^{-\varepsilon t} \mathbb{E} \int_{0}^{t} e^{\varepsilon s} N_{1}^{*}(x(s)) d s \\
\quad+e^{-\varepsilon t} \frac{\alpha \overline{\mathcal{\kappa}} p}{\alpha+p} \mathbb{E} \int_{0}^{t} e^{\varepsilon s}\left[|x(s-\tau)|^{\alpha+p}-e^{\varepsilon \tau}|x(s)|^{\alpha+p}\right] d s \\
\quad+e^{-\varepsilon t} \frac{\delta_{2} \alpha^{\prime \prime}}{\alpha^{\prime \prime}+p} \mathbb{E} \int_{0}^{t} e^{\varepsilon s}\left[|x(s-\tau)|^{\alpha^{\prime \prime}+p}-e^{\varepsilon \tau}|x(s)|^{\alpha^{\prime \prime}+p}\right] d s,
\end{aligned}
$$

where

$$
\begin{aligned}
& N_{1}^{*}(x) \\
& =\frac{p(p-1)}{2} \sigma^{2}|x|^{2 \beta+p}-\delta_{1}|x|^{\alpha^{\prime}+p}+p\left(\kappa+\frac{\bar{\kappa} p}{\alpha+p}\right)|x|^{\alpha+p} \\
& \quad+\frac{\delta_{2} p}{\alpha^{\prime \prime}+p}|x|^{\alpha^{\prime \prime}+p}+p \frac{e^{\varepsilon \tau} \bar{\kappa} \alpha}{\alpha+p}|x|^{\alpha+p}+\frac{e^{\varepsilon \tau} \delta_{2} \alpha^{\prime \prime}}{\alpha^{\prime \prime}+p}|x|^{\alpha^{\prime \prime}+p} \\
& \\
& +(p \gamma+\delta+\varepsilon)|x|^{p},
\end{aligned}
$$

$$
\begin{gathered}
\int_{0}^{t} e^{\varepsilon s}\left[|x(s-\tau)|^{\alpha^{\prime \prime}+p}-e^{\varepsilon \tau}|x(s)|^{\alpha^{\prime \prime}+p}\right] d s \\
\leq \int_{-\tau}^{0} e^{\varepsilon(s+\tau)}|\xi(s)|^{\alpha^{\prime \prime}+p} d s<\infty .
\end{gathered}
$$


Because of $2 \beta \leq \alpha<\alpha^{\prime}, \alpha^{\prime \prime}<\alpha^{\prime}$, the coefficient of polynomial in (38) is negative, it follows boundedness of $N_{1}^{*}(x)$. Repeating the procedure of Lemma 9, we have from (37) and (38) that

$$
\begin{aligned}
\mathbb{E} V(x) \leq & e^{-\varepsilon t} V(\xi(0))+\frac{\bar{N}_{1}^{*}}{\varepsilon}\left(1-e^{-\varepsilon t}\right) \\
& +e^{-\varepsilon t} \frac{\alpha \bar{\kappa} p}{\alpha+p} \int_{-\tau}^{0} e^{\varepsilon(s+\tau)}|\xi(s)|^{\alpha+p} d s \\
& +e^{-\varepsilon t} \frac{\delta_{2} \alpha^{\prime \prime}}{\alpha^{\prime \prime}+p} \int_{-\tau}^{0} e^{\varepsilon(s+\tau)}|\xi(s)|^{\alpha^{\prime \prime}+p} d s ;
\end{aligned}
$$

the rest of proof can be completed. Omit it here.

This means that the solution is bounded in the pth moment; stochastically ultimate boundedness will follow directly. It shows that the solution trajectory is bounded with large probability.

Theorem 10. The solution of (4) is stochastically ultimately bounded under the condition of Lemma 9 or Lemma 9'; that is, for any $\epsilon \in(0,1)$, there is a positive constant $\chi(=\chi(\epsilon))$ such that for any positive initial value, the solution of (4) has the property that

$$
\limsup _{t \rightarrow+\infty} P\{|x(t)|>\chi\}<\epsilon .
$$

Proof. This can be easily verified by Chebyshev's inequality and Lemma 9 or Lemma $9^{\prime}$, by choosing $\chi=\left(K_{p} / \epsilon\right)^{1 / p}$ to be sufficiently large because of the following

$$
P(|x(t)|>\chi) \leq \frac{\mathbb{E}\left[|x|^{p}\right]}{\chi^{p}} .
$$

Hence,

$$
\limsup _{t \rightarrow+\infty} P(|x(t)| \leq \chi) \geq 1-\frac{\limsup _{t \rightarrow+\infty} \mathbb{E}\left[|x|^{p}\right]}{\chi^{p}} \geq 1-\varepsilon
$$

as required.

\section{Stabilization of Noises}

From Sections 2 and 3, we know that both the Brownian noises and jump processes can suppress the potential explosion of the solution and guarantee this global solution to be bounded in the sense of the pth moment. This section is devoted to considering the stable effect of noises under some appropriate conditions; we show that jump processes may stabilize the given unstable nonlinear delay system $\dot{x}(t)=$ $f(x(t), x(t-\tau), t)$.

Theorem 11. Suppose that for the initial data $\xi \in C([-\tau, 0]$; $R^{n}$ ) satisfying $x(0) \neq 0$, the solution $x(t)$ of SDE (4) is $P(x(t) \neq 0, t \geq 0)=1$. Under Assumptions 1, 2, and 4, if, for $\sigma \neq 0$, the solution $x(t)$ of SDE (4) with any initial data $\xi \in C\left([-\tau, 0] ; R^{n}\right)$ satisfying $x(0) \neq 0$ has the property

$$
\limsup _{t \rightarrow \infty} \frac{1}{t} \log |x(t)| \leq 0 \quad \text { a.s. }
$$

Proof. By Theorem 3, suppose that almost all sample paths of $x(t)$ of (4) starting from a nonzero state will never reach the origin for all $t \geq 0$. Applying the Itô formula to the function $e^{t} \log |x(t)|$ leads to

$$
\begin{aligned}
& e^{t} \log |x(t)| \\
& =\log |x(0)| \\
& +\int_{0}^{t} e^{s}\left\{\log |x(s)|+|x(s)|^{-2}\langle x(s), f(x(s), y(s), s)\rangle\right. \\
& \quad-\frac{1}{2}\left[\sigma^{2}|x(s)|^{2 \beta}+q^{2}|x(s-\tau)|^{2 \beta^{\prime}}\right] d s \\
& \left.\quad+\int_{\mathbb{Y}}[\ln |1+H(x, y, u)|-H(x, y, u)] \lambda(d u)\right\} d s \\
& +\int_{0}^{t} e^{s} \sigma|x(s)|^{\beta} d W_{1}(s)+\int_{0}^{t} e^{s} q|x(s-\tau)|^{\beta^{\prime}} d W_{2}(s) \\
& +\int_{0}^{t} \int_{\mathbb{Y}} e^{s} \ln |1+H(x, y, u)| \widetilde{N}(d s, d u) .
\end{aligned}
$$

By virtue of exponential martingale inequality with jumps [7, Theorem 5.2.9, page 291].

$$
\begin{aligned}
& \mathbb{P}\left\{\sup _{0 \leq t \leq T}\right. {\left[\int_{0}^{t} e^{s} \sigma|x(s)|^{\beta} d W_{1}(s)-\frac{1}{2 e^{k}} \int_{0}^{t} e^{2 s} \sigma^{2}|x(s)|^{2 \beta} d s\right.} \\
&+\int_{0}^{t} \int_{\mathbb{V}} e^{s} \ln |1+H(x, y, u)| \widetilde{N}(d s, d u) \\
&-\frac{1}{e^{-k}} \int_{0}^{t} \int_{\mathbb{Y}}\left(e^{e^{s-k} \ln |1+H(x, y, u)|}-1\right. \\
&\left.\left.-e^{s-k} \ln |1+H(x, y, u)|\right) \lambda(d u) d s\right] \\
&\left.\geq 2 e^{k} \ln k\right\} \leq k^{-2} .
\end{aligned}
$$

Choose $T=k$, and $k \in \mathbb{N}$ in the above equation. Since $\sum_{\infty}^{k=1} k^{-2} \leq \infty$, by the Borel-Cantelli lemma, there exists $\Omega_{0} \subset$ $\Omega$ with $\mathbb{P}\left(\Omega_{0}\right)=1$ such that for any $\omega \in \Omega_{0}$, there exists an integer $k=k(\omega)$ that can be found satisfying

$$
\begin{aligned}
& \int_{0}^{t} e^{s} \sigma|x(s)|^{\beta} d W_{1}(s) \\
& +\int_{0}^{t} \int_{\mathbb{Y}} e^{s} \ln |1+H(x, y, u)| \widetilde{N}(d s, d u) \\
& \leq 2 e^{k} \ln k+\frac{e^{-k}}{2} \int_{0}^{t} e^{2 s} \sigma^{2}|x(s)|^{2 \beta} d s \\
& +\frac{1}{e^{-k}} \int_{0}^{t} \int_{\mathbb{Y}}\left(|1+H(x, y, u)|^{e^{s-k}}-1\right. \\
& \left.\quad-e^{s-k} \ln |1+H(x, y, u)|\right) \lambda(d u) d s
\end{aligned}
$$

whenever $k \geq k(\omega), k-1 \leq t \leq k$. 
Next, for any $\omega \in \Omega_{0}$ and $k \geq k(\omega)$, we still make use of exponential martingale inequality, (choose $\alpha=e^{-k}, \beta=$ $\left.2 e^{k} \ln k\right)$

$$
\begin{aligned}
\mathbb{P}\left\{\sup _{0 \leq t \leq T}\right. & {\left[\int_{0}^{t} e^{s} q|x(s-\tau)|^{\beta^{\prime}} d W_{2}(s)\right.} \\
- & \left.\left.\frac{1}{2 e^{\kappa}} \int_{0}^{t} e^{2 s} q^{2}|x(s-\tau)|^{2 \beta^{\prime}} d s\right] \geq 2 e^{\kappa} \ln \kappa\right\} \leq \kappa^{-2} .
\end{aligned}
$$

Thus, for all $\omega \in \Omega_{0}$ and $k-1<s<t \leq k, k \geq k(\omega)$, we also have

$$
\begin{aligned}
\int_{0}^{t} e^{s} q|x(s-\tau)|^{\beta^{\prime}} d W_{2}(s) \\
\leq 2 e^{k} \ln k+\frac{1}{2 e^{k}} \int_{0}^{t} e^{2 s} q^{2}|x(s-\tau)|^{2 \beta^{\prime}} d s \\
\leq 2 e^{k} \ln k+\frac{1}{2} \int_{0}^{t} e^{s} q^{2}|x(s-\tau)|^{2 \beta^{\prime}} d s .
\end{aligned}
$$

In the following, we divide two cases.

(1) If $\alpha>2 \beta$, noting inequality $x^{r} \leq 1+r(x-1), x \geq 0$, $0 \leq r \leq 1$, and $e^{s-k}<1$ for $\omega \in \Omega_{0}$ and $k-1 \leq s \leq t \leq$ $k, k \geq k_{0}(\omega)$, substituting (47)-(49) into (45) gives

$$
\log |x(t)|
$$

$$
\begin{aligned}
& \leq e^{-t} \log x(0)+4 e^{k-t} \ln k \\
& +\int_{0}^{t} e^{s-t}\left[\log |x(s)|-\frac{\sigma^{2}\left(1-e^{s-k}\right)}{2}|x(s)|^{2 \beta}\right. \\
& \left.+\kappa|x(s)|^{\alpha}+\bar{\kappa}|y(s)|^{\alpha}+\gamma\right] d s \\
& +\frac{1}{e^{-k}} \int_{0}^{t} e^{-t} \int_{\mathbb{Y}}\left(|1+H(x, y, u)|^{e^{s-k}}\right. \\
& \left.-1-e^{s-k}|H(x, y, u)|\right) \lambda(d u) d s \\
& \leq e^{-t} \log x(0)+4 e^{k-t} \ln k+\bar{\kappa} \int_{-\tau}^{0} e^{s+\tau}|\xi(s)|^{\alpha} d s \\
& +\int_{0}^{t} e^{s-t}\left[-\frac{\sigma^{2}\left(1-e^{s-k}\right)}{2}|x(s)|^{2 \beta}+\left(\kappa+\bar{\kappa} e^{\tau}\right)|x(s)|^{\alpha}\right. \\
& +\gamma+\log |x(s)|] d s,
\end{aligned}
$$

where

$$
\phi(x)=:-\frac{\sigma^{2}\left(1-e^{s-k}\right)}{2}|x|^{2 \beta}+\left(\kappa+\bar{\kappa} e^{\tau}\right)|x|^{\alpha}+\gamma+\log |x| .
$$

Also, $\alpha>2 \beta$ means that there exists a constant $M_{1}$ such that $\phi(x) \leq M_{1}$, which leads to

$$
\begin{aligned}
\log |x(t)| \leq & e^{-t} \log x(0)+\bar{\kappa} \int_{-\tau}^{0} e^{s+\tau}|\xi(s)|^{\alpha}+4 e^{k-t} \ln k \\
& +M_{1} \int_{0}^{t} e^{s-t} d s
\end{aligned}
$$

$$
\begin{aligned}
& \frac{\log |x(t)|}{\ln t} \\
& \leq \frac{1}{\ln (k-1)}\left[e^{-t} \log x(0)+\bar{\kappa} \int_{-\tau}^{0} e^{s+\tau}|\xi(s)|^{\alpha} d s+M_{1}\right] \\
& \quad+\frac{4 e \ln k}{\ln (k-1)} \text { a.s. }
\end{aligned}
$$

It follows that

$$
\limsup _{t \rightarrow \infty} \frac{\log |x(t)|}{\ln t} \leq 4 e .
$$

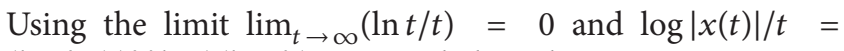
$(\log |x(t)| / \ln t)(\ln t / t)$, we can deduce that

$$
\limsup _{t \rightarrow \infty} \frac{\log |x(t)|}{t} \leq 0, \quad \text { a.s. }
$$

Otherwise, we consider that

(2) if $2 \beta \leq \alpha<\alpha^{\prime}$ and $\alpha^{\prime \prime}<\alpha^{\prime}$, we obtain

$\log |x(t)|$

$$
\begin{aligned}
& \leq e^{-t} \log x(0)+4 e^{k-t} \ln k+\bar{\kappa} \int_{-\tau}^{0} e^{s+\tau}|\xi(s)|^{\alpha} d s \\
& +\int_{0}^{t} e^{s-t}\left[-\frac{\sigma^{2}\left(1-e^{s-k}\right)}{2}|x(s)|^{2 \beta}+\left(\kappa+\bar{\kappa} e^{\tau}\right)|x(s)|^{\alpha}\right. \\
& \quad+\gamma+\log |x(s)|] d s \\
& +\int_{0}^{t} e^{s-t}\left[\frac{1}{e^{s-k}}\left(\delta-\delta_{1}|x(s)|^{\alpha^{\prime}}+\delta_{2}|y(s)|^{\alpha^{\prime \prime}}\right)\right] d s \\
& \leq e^{-t} \log x(0)+4 e^{k-t} \ln k+\bar{\kappa} \int_{-\tau}^{0} e^{s+\tau}|\xi(s)|^{\alpha} d s \\
& +\delta_{2} \int_{-\tau}^{0}|\xi(s)|^{\alpha^{\prime \prime}} d s \\
& +\int_{0}^{t} e^{s-t}\left[-\frac{\sigma^{2}\left(1-e^{s-k}\right)}{2}|x(s)|^{2 \beta}+\left(\kappa+\bar{\kappa} e^{\tau}\right)|x(s)|^{\alpha}\right.
\end{aligned}
$$

$$
\begin{gathered}
+\gamma+\log |x(s)|] d s \\
+\int_{0}^{t} e^{s-t}\left[\frac{1}{e^{s-k}}\left(\delta-\delta_{1}|x(s)|^{\alpha^{\prime}}+\delta_{2}|x(s)|^{\alpha^{\prime \prime}}\right)\right] d s \\
\leq e^{-t} \log x(0)+4 e^{k-t} \ln k+\bar{\kappa} \int_{-\tau}^{0} e^{s+\tau}|\xi(s)|^{\alpha} d s \\
+\delta_{2} \int_{-\tau}^{0}|\xi(s)|^{\alpha^{\prime \prime}} d s+\int_{0}^{t} e^{s-t}[\phi(x)+J(x)] d s,
\end{gathered}
$$


where

$$
\begin{gathered}
\phi(x)=:-\frac{\sigma^{2}\left(1-e^{s-k}\right)}{2}|x|^{2 \beta}+\left(\kappa+\bar{\kappa} e^{\tau}\right)|x|^{\alpha}+\gamma+\log |x|, \\
J(x)=: \frac{1}{e^{s-k}}\left(\delta-\delta_{1}|x|^{\alpha^{\prime}}+\delta_{2}|x|^{\alpha^{\prime \prime}}\right) .
\end{gathered}
$$

Noting that for $\omega \in \Omega_{0}$ and $k-1 \leq s \leq t \leq k, k \geq k_{0}(\omega)$, we know that $\phi(x)+J(x)$ is bounded $M_{2}$ by a polynomial with negative leading term. Note that

$$
\begin{aligned}
& \log |x(t)| \leq e^{-t} \log x(0)+\bar{\kappa} \int_{-\tau}^{0} e^{s+\tau}|\xi(s)|^{\alpha} d s \\
& +\delta_{2} \int_{-\tau}^{0}|\xi(s)|^{\alpha^{\prime \prime}} d s+4 e^{k-t} \ln k+M_{2} \int_{0}^{t} e^{s-t} d s, \\
& \leq \frac{1}{\ln (k-1)} \\
& \times\left(e^{-t} \log x(0)+\bar{\kappa} \int_{-\tau}^{0} e^{s+\tau}|\xi(s)|^{\alpha} d s\right. \\
& \left.+\delta_{2} \int_{-\tau}^{0}|\xi(s)|^{\alpha^{\prime \prime}} d s+M_{2}\right)+\frac{4 e \ln k}{\ln (k-1)} \text { a.s. }
\end{aligned}
$$

We conclude that

$$
\limsup _{t \rightarrow \infty} \frac{\log |x(t)|}{t} \leq 0, \quad \text { a.s. }
$$

Thus, assertion (44) follows.

In order to highlight the stable effect of Lévy noise, let

$$
H\left(x\left(t^{-}\right), x(t-\tau)^{-}, u\right)=H^{\prime}(t, u) ;
$$

we will discuss almost sure exponentially stability of the following SDE with jumps:

$$
\begin{aligned}
d x(t)= & f(x(t), x(t-\tau), t) d t+\sigma|x(t)|^{\beta} x(t) d W_{1}(t) \\
& +q x(t)|x(t-\tau)|^{\beta^{\prime}} d W_{2}(t) \\
& +x(t) \int_{\mathbb{Y}} H^{\prime}(t, u) \widetilde{N}(d t, d u) .
\end{aligned}
$$

Assumption 12. For any $t \geq 0, u \in \mathbb{Y}$, there exists a constant $C>0$ such that

$$
\int_{\mathbb{Y}}\left[\ln \left(1+H^{\prime}(t, u)\right)\right]^{2} \lambda(d u) \leq C .
$$

Theorem 13. Suppose the initial data $\xi \in C\left([-\tau, 0] ; R^{n}\right)$ satisfy $\xi(0) \neq 0$; then the solution $x(t)$ of SDE (60) is such that $x(t) \neq 0$ a.s. for all $t \geq 0$. Under Assumptions 1, 2, and 12, if for $\epsilon \epsilon$ $(0,1)$ and $2 \beta>\alpha, \sigma \neq 0$, the solution $x(t)$ of SDE (60) has the property

$$
\limsup _{t \rightarrow \infty} \frac{1}{t} \log |x(t)| \leq(\phi-\bar{H}) \quad \text { a.s., }
$$

where

$$
\begin{gathered}
\phi=\max _{x \in R^{n}}\left\{-\frac{\sigma^{2}}{2}|x|^{2 \beta}+(\kappa+\bar{\kappa})|x|^{\alpha}+\gamma\right\}, \\
\bar{H}=\min _{t \geq 0} \int_{\mathbb{Y}}\left[H^{\prime}(t, u)-\ln \left|1+H^{\prime}(t, u)\right|\right] \lambda(d u) .
\end{gathered}
$$

In particular, nonlinear delay system (60) is a.s. exponentially stable if $\phi-\bar{H}<0$.

Proof. By Theorem 3 and supposing $x(t) \neq 0$ a.s., if the initial data $\xi \in C\left([-\tau, 0] ; R^{n}\right)$ satisfying $x(0) \neq 0$, the solution $x(t)$ with a given initial value will exist a.s. for all $t \geq 0$. Applying the Itô formula to the function $\log |x(t)|$ leads to

$$
\begin{aligned}
\log |x(t)|= & \log |x(0)| \\
& +\int_{0}^{t}|x(s)|^{-2}\langle x(s), f(x(s), x(x-\tau), s)\rangle \\
& -\frac{1}{2}\left[\sigma^{2}|x(s)|^{2 \beta}+q^{2}|x(s-\tau)|^{2 \beta^{\prime}}\right] d s \\
& -\int_{0}^{t} \int_{\mathbb{V}}\left[H^{\prime}(s, u)-\ln \left|1+H^{\prime}(s, u)\right|\right] \lambda(d u) d s \\
& +\int_{0}^{t} \sigma|x(s)|^{\beta} d W_{1}(s)+\int_{0}^{t} q|x(s-\tau)|^{\beta^{\prime}} d W_{2}(s) \\
& +\int_{0}^{t} \int_{\mathbb{V}} \ln \left|1+H^{\prime}(s, u)\right| \widetilde{N}(d s, d u),
\end{aligned}
$$

where

$$
\begin{gathered}
M_{1}(t)=\int_{0}^{t} \sigma|x(s)|^{\beta} d W_{1}(s), \\
M_{2}(t)=\int_{0}^{t} q|x(s-\tau)|^{\beta} d W_{2}(s), \\
M_{3}(t)=\int_{0}^{t} \int_{\mathbb{V}} \ln \left|1+H^{\prime}(s, u)\right| \widetilde{N}(d s, d u) .
\end{gathered}
$$

By virtue of exponential martingale inequality [4, Theorem 7.4, page 44].

$$
\begin{gathered}
\mathbb{P}\left\{\sup _{0 \leq t \leq T}\left[\int_{0}^{t} \sigma|x(s)|^{\beta} d W_{1}(s)-\frac{\epsilon}{2} \int_{0}^{t} \sigma^{2}|x(s)|^{2 \beta} d s\right]\right. \\
\geq \xi \ln \kappa\} \leq \kappa^{-\epsilon \xi} .
\end{gathered}
$$

Choose $T=\kappa$, and $\kappa \in \mathbb{N}, 0<\epsilon<1, \gamma>0$, and $\epsilon \xi>1$ in the above equation. Since $\sum_{\infty}^{\kappa=1} \kappa^{-\epsilon \xi} \leq \infty$, by the BorelCantelli lemma, there exists an $\Omega_{0} \subset \Omega$ with $\mathbb{P}\left(\Omega_{0}\right)=1$ such 
that, for any $\omega \in \Omega_{0}$, there exists an integer $\kappa=\kappa(\omega, \epsilon)$ that can be found such that

$$
\int_{0}^{t} \sigma|x(s)|^{\beta} d W_{1}(s) \leq \xi \ln \kappa+\frac{\epsilon}{2} \int_{0}^{t} \sigma^{2}|x(s)|^{2 \beta} d s .
$$

Whenever $\kappa \geq \kappa(\omega, \epsilon), \kappa-1 \leq t \leq \kappa$ a.s. Similarly, we can obtain

$$
\int_{0}^{t} q|x(s-\tau)|^{\beta^{\prime}} d W_{2}(s) \leq 2 \ln \kappa+\frac{1}{2} \int_{0}^{t} q^{2}|x(s-\tau)|^{2 \beta^{\prime}} d s .
$$

Whenever $\kappa \geq \kappa(\omega, \epsilon), \kappa-1 \leq t \leq \kappa$ a.s.

In other words, we have shown that

$$
\begin{aligned}
& \log |x(t)| \\
& \leq \log |x(0)|+\xi \ln \kappa+2 \ln \kappa \\
& +\int_{0}^{t}\left[-\frac{\sigma^{2}(1-\epsilon)}{2}|x(s)|^{2 \beta}+\kappa|x(s)|^{\alpha}+\bar{\kappa}|y(s)|^{\alpha}+\gamma\right] d s \\
& -\int_{0}^{t} \int_{\mathbb{Y}}\left[H^{\prime}(s, u)-\ln \left|1+H^{\prime}(s, u)\right|\right] \lambda(d u) d s \\
& +\int_{0}^{t} \int_{\mathbb{Y}} \ln \left|1+H^{\prime}(s, u)\right| \widetilde{N}(d s, d u) \\
& \leq \log ^{2}|x(0)|+\xi \ln \kappa+2 \ln \kappa+\bar{\kappa} \int_{-\tau}^{0}|\xi(s)|^{\alpha} d s \\
& +\int_{0}^{t}\left[-\frac{\sigma^{2}(1-\epsilon)}{2}|x(s)|^{2 \beta}+(\kappa+\bar{\kappa})|x(s)|^{\alpha}+\gamma\right] d s \\
& +\int_{0}^{t}\left[\phi_{\epsilon}-\bar{H}\right] d s+\int_{0}^{t} \int_{\mathbb{Y}} \ln \left|1+H^{\prime}(s, u)\right| \widetilde{N}(d s, d u), \\
& +\int_{0}^{t}\left[H_{\mathbb{Y}}^{\prime}(s, u)-\ln \left|1+H^{\prime}(s, u)\right|\right] \lambda(d u) d s \\
& +\int_{0}^{t} \int_{\mathbb{Y}} \ln \left|1+H^{\prime}(s, u)\right| \widetilde{N}(d s, d u) \\
& +\log |x(0)|+\xi \ln \kappa+2 \ln \kappa+\bar{\kappa} \int_{-\tau}^{0}|\xi(s)|^{\alpha} d s
\end{aligned}
$$

where

$$
\begin{aligned}
& \phi_{\epsilon}=: \max _{x \in R^{n}}\left(-\frac{\sigma^{2}(1-\epsilon)}{2}|x|^{2 \beta}+(\kappa+\bar{\kappa})|x|^{\alpha}+\gamma\right), \\
& \bar{H}=: \min _{t \geq 0} \int_{\mathbb{Y}}\left[H^{\prime}(t, u)-\ln \left|1+H^{\prime}(t, u)\right|\right] \lambda(d u) .
\end{aligned}
$$

Next, noting Assumption 12, for any $\omega \in \Omega_{0}$, and $0<\epsilon<$ $1, \kappa-1 \leq t \leq \kappa$ with $\kappa \geq \kappa(\omega, \epsilon)$

$$
\left\langle M_{3}(t), M_{3}(t)\right\rangle_{t}=\int_{0}^{t} \int_{\mathbb{Y}}\left|1+H^{\prime}(s, u)\right|^{2} \lambda(d u) d s \leq C t .
$$

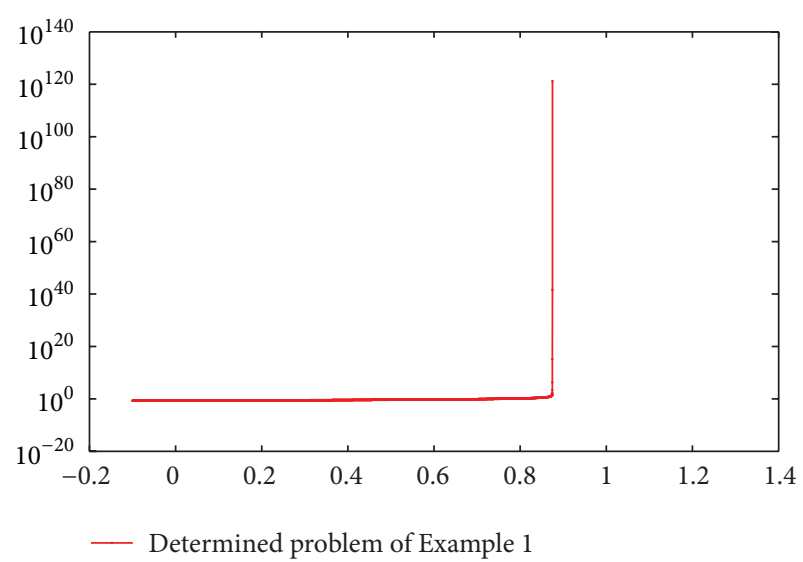

FIGURE 2: We simulate the discrete equations for delay equation (77).

Then the strong law of large numbers yields

$$
\lim _{t \rightarrow \infty} \frac{1}{t} \int_{0}^{t} \int_{\mathbb{Y}} \ln \left|1+H^{\prime}(s, u)\right| \widetilde{N}(d s, d u)=0
$$

Thus, for $\omega \in \Omega_{0}$ and $(\kappa-1) \leq t \leq \kappa, \epsilon \rightarrow 0$, it follows from (69) and (72) that

$$
\limsup _{t \rightarrow \infty} \frac{1}{t} \log |x(t)| \leq \phi-\bar{H} \quad \text { a.s. }
$$

Clearly, if $\phi-\bar{H}<0$, system (60) is a.s. exponentially stable; the proof is completed.

Remark 14. Compared with results in [1], authors discussed the SDE:

$$
\begin{aligned}
d x(t)=f & (x(t), x(t-\delta(t)), t) d t \\
& +q x(t) d W_{1}(t)+\sigma|x(t)|^{\beta} x(t) d W_{2}(t) ;
\end{aligned}
$$

the results show that polynomial Brownian noise $\sigma|x(t)|^{\beta} x(t) d W_{2}(t)$ can suppress this potential explosion and another linear Brownian noise $q x(t) d W_{1}(t)$ has an effect to stabilize the suppressed equation.

Theorem 13 shows that under Assumptions 1, 2, and 12, choosing appropriate function $H$, together with $2 \beta>\alpha$, $\sigma \neq 0$, Brownian noises may suppress the given deterministic equation $\dot{x}(t)=f(x(t), x(t-\tau), t)$. Linear jump process has stable effect on system (60); it has the same role as linear Brownian noise.

Example 1. Consider stochastic differential equation

$$
\begin{aligned}
d x(t)= & x(t)\left[1+2 x^{2}(t)+6 x^{2}(t-\tau)\right] d t+2 x^{3}(t) d W_{1}(t) \\
& +2 x^{2}(t-\tau) x(t) d W_{2}(t) \\
& +x(t) \int_{\mathbb{V}} H^{\prime}(u) \widetilde{N}(d t, d u),
\end{aligned}
$$




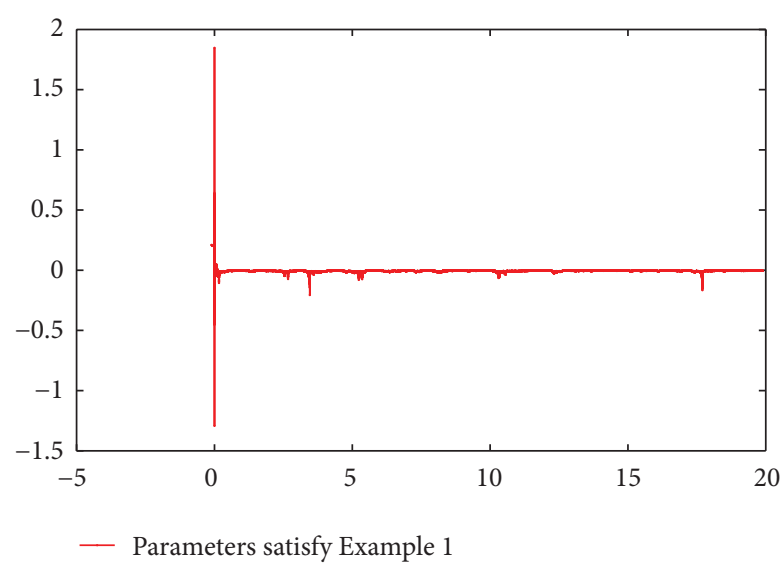

FIGURE 3: We simulate the discretization equations for stochastic delay equation (75), where $\sigma=2, \bar{\kappa}=6, \kappa=2, \gamma=1, \alpha=\beta=2$, $\beta^{\prime}=2$, and $H^{\prime}=0.1 e^{-u^{2} / 2}$ such that $[\phi-\bar{H}]<0$.

where $q=2, \sigma=2, \bar{\kappa}=6, \kappa=2, \gamma=1, \alpha=\beta=2, \beta^{\prime}=2$,

$$
\begin{gathered}
\phi=\max _{x \in R, x \neq 0}\left\{-2 x^{4}+8 x^{2}+1\right\}=9, \\
\bar{H}=\int_{\mathbb{Y}}\left[H^{\prime}(u)-\ln \left|1+H^{\prime}(u)\right|\right] \lambda(d u) .
\end{gathered}
$$

Figure 2 shows the differential equation

$$
\frac{d x(t)}{d t}=x(t)\left[1+2 x^{2}(t)+6 x^{2}(t-\tau)\right] .
$$

Simulation indicates that the solution of (78) is explosive in finite time. See Figure 2.

If we choose function $H^{\prime}=0.1 e^{-u^{2} / 2}$ such that $[\phi-\bar{H}]<$ 0 , we can conclude that system (75) is a.s. exponentially stable by Theorems 13 . See Figure 3.

But we cannot indicate that system (75) is a.s. exponentially stable if we choose function $H^{\prime}$ such that $[\phi-\bar{H}]>0$, although it has global solution. See Figure 4.

Example 2. Consider another stochastic differential equation

$$
\begin{aligned}
d x(t)= & x(t)\left[1+2 x^{3}(t)+2 x^{3}(t-\tau)\right] d t \\
& +0.1 x^{3}(t) d W_{1}(t)+3 x(t-\tau) x(t) d W_{2}(t) \\
& +x(t) \int_{\mathbb{V}} H^{\prime}(u) \widetilde{N}(d t, d u)
\end{aligned}
$$

where $q=3, \sigma=0.1, \bar{\kappa}=2, \kappa=2, \gamma=1, \alpha=\beta=3, \beta^{\prime}=1$,

$$
\begin{gathered}
\phi=\max _{x \in R, x \neq 0}\left\{-\frac{1}{200} x^{6}+4 x^{3}+1\right\}, \\
\bar{H}=\int_{\mathbb{Y}}\left[H^{\prime}(u)-\ln \left|1+H^{\prime}(u)\right|\right] \lambda(d u) .
\end{gathered}
$$

Figure 5 shows that the corresponding determined delay equation of (78) is explosive in finite time.

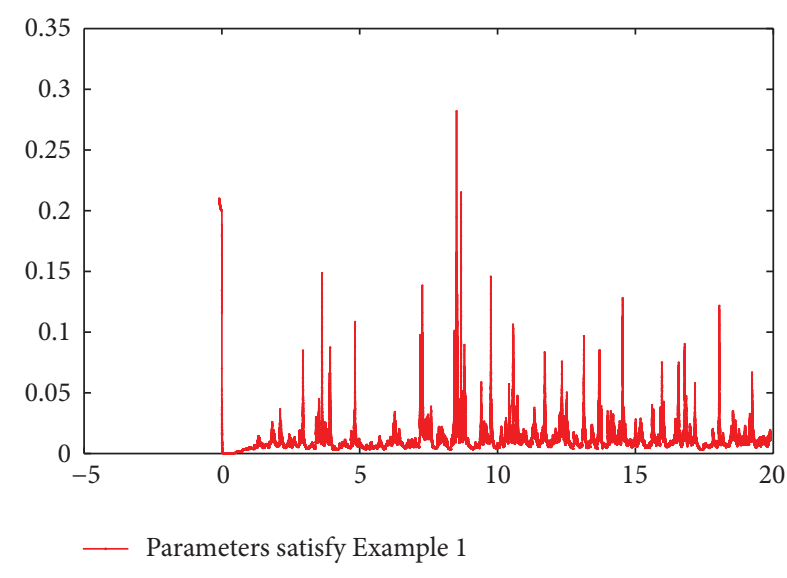

FIgURE 4: We simulate the discretization equations for stochastic delay equation (75); $H^{\prime}=10 e^{-u^{2} / 2}$ such that $[\phi-\bar{H}]>0$.

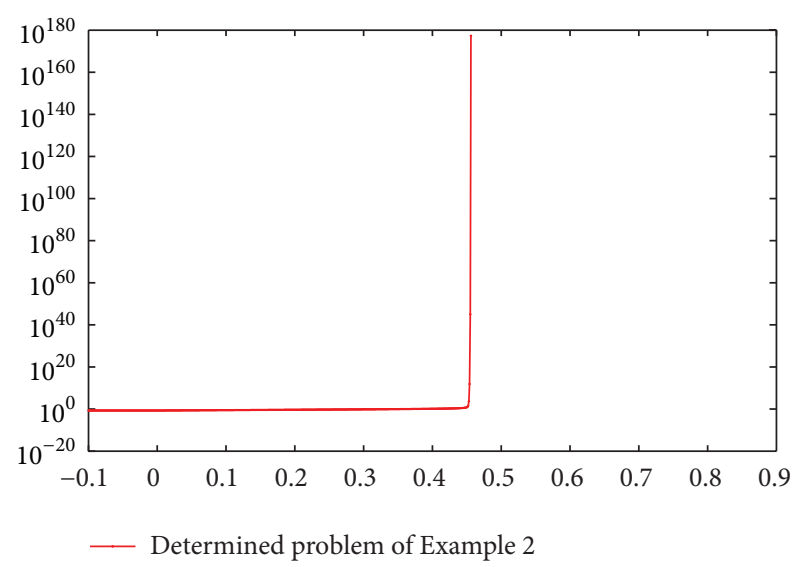

FIGURE 5: We simulate the discretization equations for determined delay equation: $d x(t) / d t=x(t)\left[1+2 x^{3}(t)+2 x^{3}(t-\tau)\right]$.

If we choose function $H^{\prime}$ such that $[\phi-\bar{H}]<0$, we can show that system (78) is a.s. exponentially stable. See Figures 6 and 7.

\section{Conclusions and Extensions}

In this paper, we consider stochastic nonlinear delay differential system with jumps, where $f$ satisfies the oneside polynomial growth condition. Our main results include the following theoretic analysis. Theorems 3 and 5 show that Brownian noises or Lévy noise may suppress potential explosion of the solution for some appropriate parameters. Using the exponential martingale inequality with jumps, we discuss that the sample Lyapunov exponent is nonpositive in Theorem 11. Lévy noise is sudden and severe environmental perturbations; if we propose linear Lévy processes in (60), by the strong law of large number for local martingale, sufficient conditions for a.s. exponentially stable are investigated. Theorem 13 reveals the stabilization property of Lévy noise.

Two interesting questions deserve further consideration and investigation. 


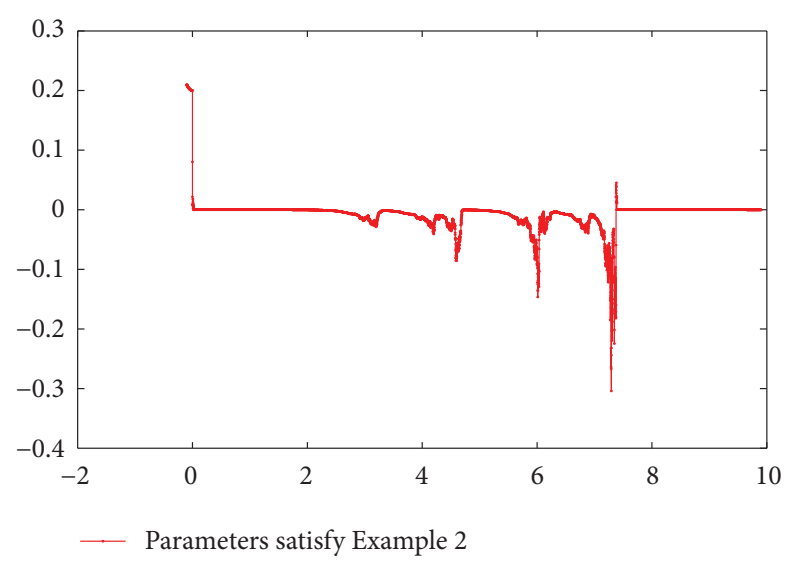

Figure 6: We simulate the discretization equations for stochastic delay equation (78), where $q=3, \sigma=0.1, \bar{\kappa}=2, \kappa=2, \gamma=1$, $\alpha=\beta=3$ and $\beta^{\prime}=1$, if we choose function $H^{\prime}=0.1 e^{-u^{2} / 2}$ such that $[\phi-\bar{H}]<0$.

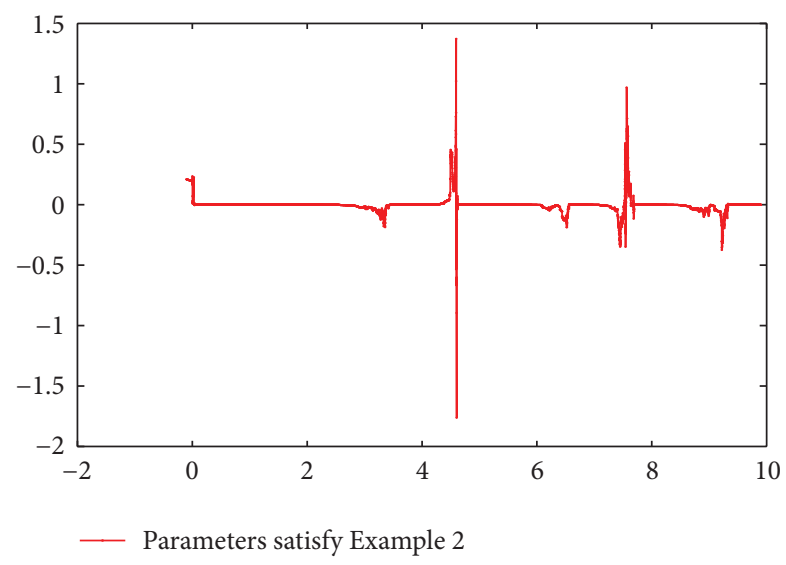

FIgURE 7: We simulate the discretization equations for stochastic delay equation (78), where $q=3, \sigma=0.1, \bar{\kappa}=2, \kappa=2, \gamma=1$, $\alpha=\beta=3$, and $\beta^{\prime}=1$. Choose function, which is different from Figure $6, H^{\prime}=e^{-u^{2} / 2}$ such that $[\phi-\bar{H}]<0$.

If we use the continuous time Markov chain $\alpha(t)$ with finite state space $\mathbb{S}=\{1,2, \ldots, N\}$ to model abrupt changes in their structure and parameters, another complex hybrid system with jumps can be studied:

$$
\begin{aligned}
d x(t)= & f(x(t), x(t-\tau), \alpha(t)) d t \\
& +\sigma(\alpha(t))|x(t)|^{\beta} x(t) d W_{1}(t) \\
& +q(\alpha(t))|x(t-\tau)|^{\beta^{\prime}} x(t) d W_{2}(t) \\
& +x(t) \int_{\mathbb{V}} H\left(x\left(t^{-}\right), x(t-\tau)^{-}, \alpha(t), u\right) \widetilde{N}(d t, d u),
\end{aligned}
$$

with initial data $\{x(t):-\tau \leq t \leq 0\}=\{\xi(t):-\tau \leq t \leq 0 \in$ $\left.C_{\mathscr{F}_{0}}^{b}([-\tau, 0])\right\}$.
We can also consider stochastic functional equation:

$$
\begin{aligned}
d x(t)= & f\left(x_{t}, \alpha(t)\right) d t+q(\alpha(t)) x(t) d W_{1}(t) \\
& +\sigma(\alpha(t))|x(t)|^{\beta} x(t) d W_{2}(t) \\
& +x(t) \int_{\mathbb{Y}} H(\alpha(t), u) \widetilde{N}(d t, d u) .
\end{aligned}
$$

The effect of suppression solution of different types of noises and stabilization of jump process to system (80) or (81) still attract our main attention. These investigations are in progress and we will report it in several articles.

\section{Conflict of Interests}

The authors declare that there is no conflict of interests regarding the publication of this paper.

\section{Acknowledgments}

This work is project supported by the National Science Foundation of China (nos. 11101183, 11171056, 11171081, 11371169, and 11271157) and 985 Program of Jilin University.

\section{References}

[1] F. Wu and S. Hu, "Stochastic suppression and stabilization of delay differential systems," International Journal of Robust and Nonlinear Control, vol. 21, no. 5, pp. 488-500, 2011.

[2] F. Wu, X. Mao, and S. Hu, "Stochastic suppression and stabilization of functional differential equations," Systems \& Control Letters, vol. 59, no. 12, pp. 745-753, 2010.

[3] F. Wu and S. Hu, "Suppression and stabilisation of noise," International Journal of Control, vol. 82, no. 11, pp. 2150-2157, 2009.

[4] X. Mao, Stochastic Differential Equations and their Applications, Ellis Horwood, Chichester, UK, 1997.

[5] C. Yuan and X. Mao, "Stability of stochastic delay hybrid systems with jumps," European Journal of Control, vol. 6, pp. 595-608, 2010.

[6] G. Yin and F. Xi, "Stability of regime-switching jump diffusions," SIAM Journal on Control and Optimization, vol. 48, no. 7, pp. 4525-4549, 2010.

[7] D. Applebaum, Lévy Processes and Stochastic Calculus, Cambridge University Press, Cambridge, UK, 2nd edition, 2009. 


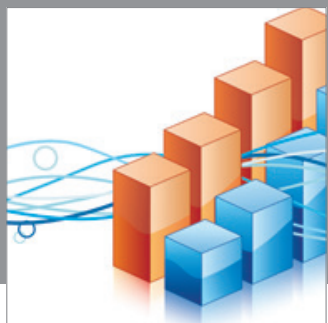

Advances in

Operations Research

mansans

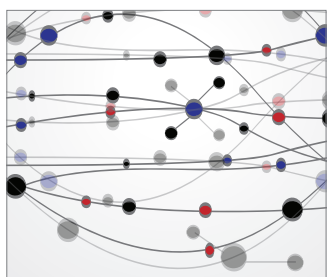

The Scientific World Journal
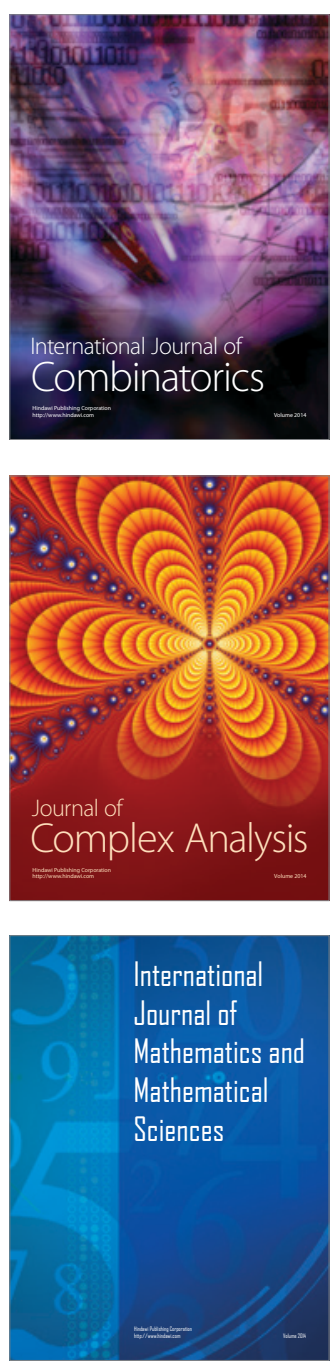
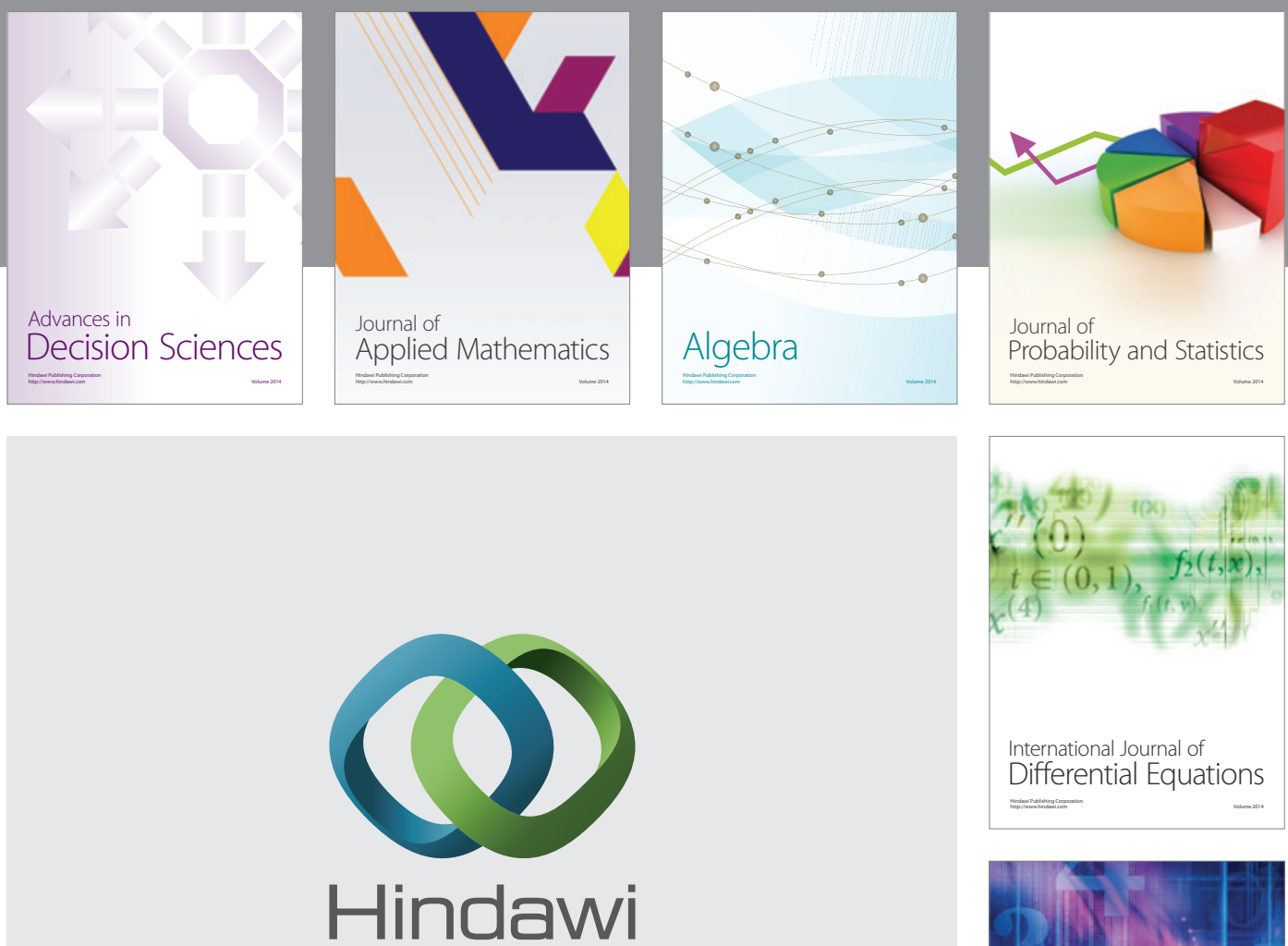

Submit your manuscripts at http://www.hindawi.com
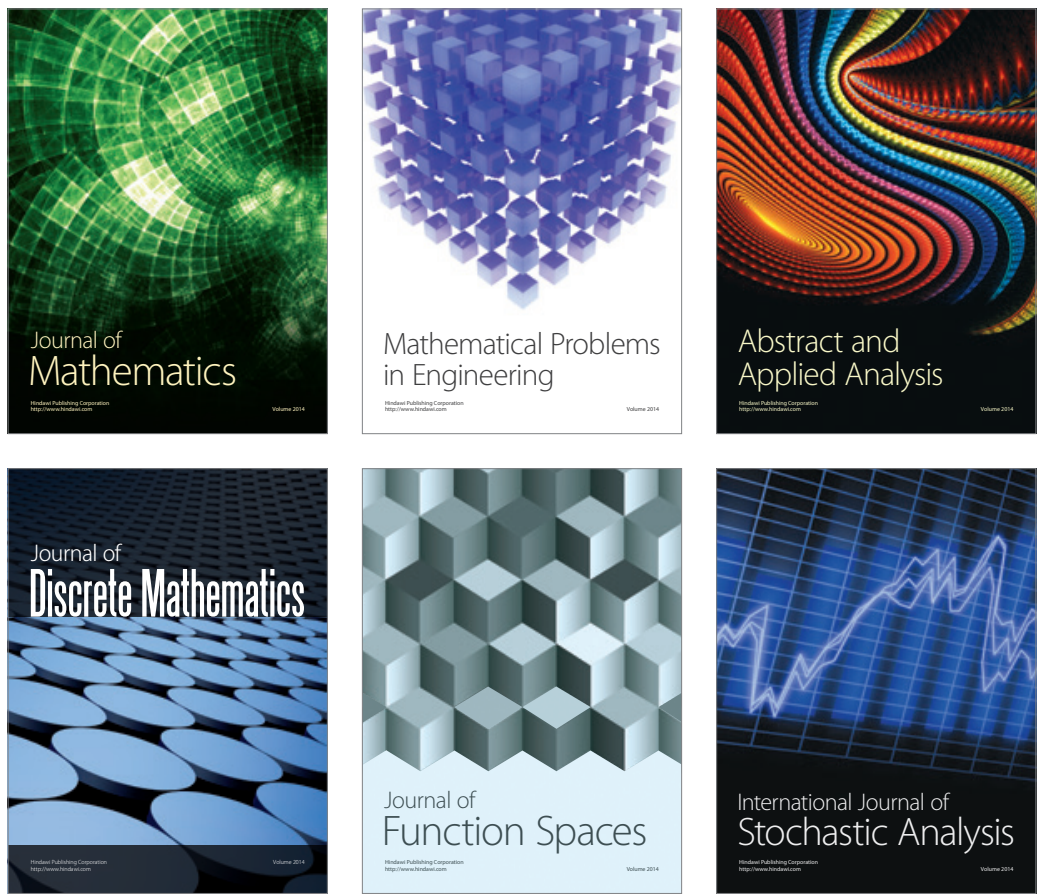

Journal of

Function Spaces

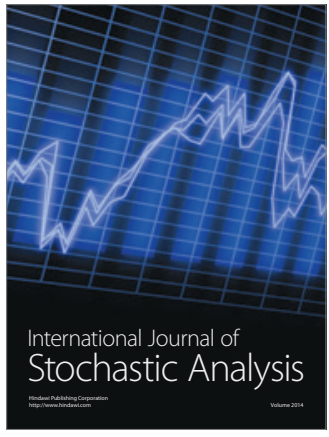

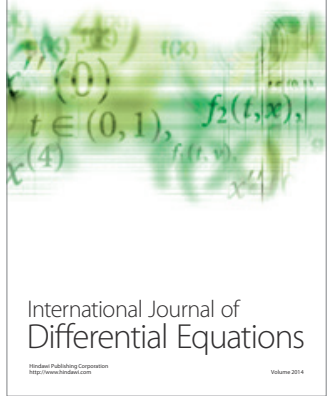
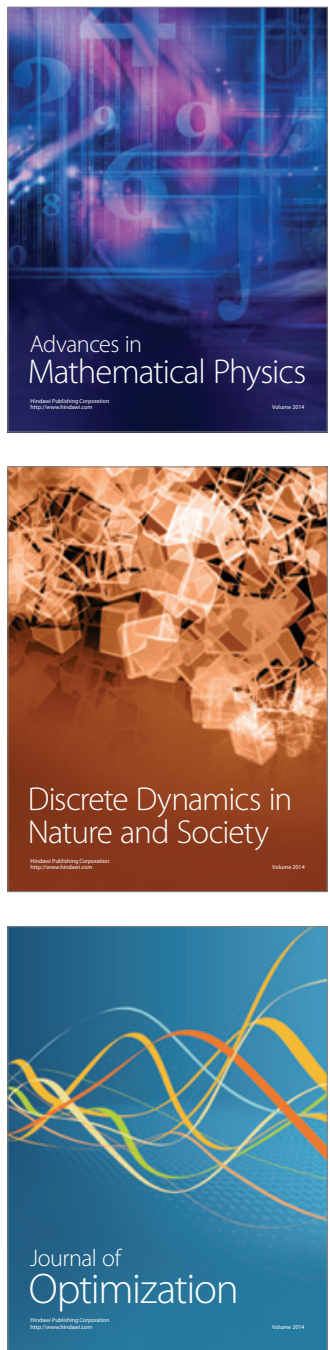